\title{
Overexpression of Tyro3 receptor tyrosine kinase leads to the acquisition of taxol resistance in ovarian cancer cells
}

\author{
CHUHEE LEE

\begin{abstract}
Department of Biochemistry and Molecular Biology, School of Medicine, Yeungnam University, Daegu 705-717, Republic of Korea
\end{abstract}

Received June 28, 2014; Accepted March 9, 2015

DOI: $10.3892 / \mathrm{mmr} .2015 .3542$

\begin{abstract}
The majority of patients with ovarian cancer are diagnosed at the advanced stages (III/IV) and their 5-year-survival rate is relatively low. One of the major causes of the poor prognosis of ovarian cancer is the development of resistance to first-line chemotherapy, including platinum and taxol. Therefore, improvements in current understanding of chemoresistance is required for the successful treatment of ovarian cancer. In the present study, taxol-resistant ovarian cancer cells, SKOV3/TR, were established by exposing parental SKOV3 cells to increasing concentrations of taxol. Briefly, cells were treated with $1.5 \mathrm{nM}$ (for 4 weeks), $3 \mathrm{nM}$ (for 4 weeks), $6 \mathrm{nM}$ (for 5 weeks), $12 \mathrm{nM}$ (for 5 weeks) and 24 nM taxol (for 8 weeks) over 6 months. The SKOV3/TR cells were found to be smaller in size and rounder in shape compared with their parental cells. Cell viability and colony formation assays demonstrated an increase in the population doubling time of the SKOV3/TR cells, indicating a reduction in the proliferative capacity of these cells. Reverse transcription-polymerase chain reaction and western blot analysis revealed that, among the TAM receptor tyrosine kinases (RTKs), the mRNA and protein expression levels of Tyro3 RTK were increased, while those of Axl and Mer RTK were decreased in the SKOV3/TR cells. In addition, restoration of the level of Tyro3 by transfecting Tyro3-specific small interfering RNA into the SKOV3/TR cells reduced the proliferative capacity of the cells, indicating that upregulation of the expression of Tyro3 in SKOV3/TR cells may promote survival in the presence of taxol, which eventually resulted in the acquisition of resistance upon taxol treatment. The present study subsequently found that, in the SKOV3/TR cells, the level of intracellular reactive oxygen species (ROS) was elevated, and
\end{abstract}

Correspondence to: Dr Chuhee Lee, Department of Biochemistry and Molecular Biology, School of Medicine, Yeungnam University, 317-1 Daemyung 5-Dong, Daegu 705-717, Republic of Korea E-mail: chlee2@ynu.ac.kr

Key words: Akt, ovarian cancer, reactive oxygen species, signal transducers and activators of transcription 3, taxol resistance, Tyro3 antioxidant treatment with $\mathrm{N}$-acetyl cysteine (NAC) exerted more profound antiproliferative effects compared with the parental cells. The western blot analysis demonstrated that treatment of the SKOV3/TR cells with NAC reduced the protein expression of Tyro3, and the inhibitory effect of NAC on the phosphorylation of Akt was increased, which may have had a positive effect on the proliferation of the SKOV3/TR cells. The levels of phosphorylation and protein expression of signal transducers and activators of transcription 3 (STAT3) were not affected by NAC treatment, indicating that the phosphorylation of Akt, but not expression or phosphorylation of STAT3, was associated with the increased intracellular ROS level in the SKOV3/TR cells. Taken together, the results of the present study demonstrated that the acquired taxol resistance of ovarian cancer cells was associated with ROS-dependent upregulation in the expression of Tyro3 RTK and the subsequent activation of Akt.

\section{Introduction}

The majority of patients with ovarian cancer $(>80 \%)$ are found to have metastasis when they are first diagnosed (1). Therefore, cytoreductive surgery and subsequent chemotherapy is common in the treatment of ovarian cancer. For the last 30 years, the chemotherapy regimens for ovarian cancer have been developed using one or a combination of cytotoxic agents, including melphalan, cyclophosphamide, doxorubicin, cisplatin, carboplatin and paclitaxel $(2,3)$. Following a long-term follow-up study by Goldberg et al, which demonstrated the significant antiproliferative effect of paclitaxel in platinum-refractory patients (4), the addition of paclitaxel to platinum-based chemotherapy has been established as a standard treatment in ovarian cancer. While the majority of patients initially respond to the first-line chemotherapy of a combination of paclitaxel and cisplatin/carboplatin, it has been reported that $\sim 30 \%$ of patients in advanced stages are unresponsive, exhibiting intrinsic chemoresistance, and initial responders also eventually acquire chemoresistance over time (5-8). These intrinsic and acquired resistances to chemotherapeutic agents have been a major obstacle in the treatment of ovarian cancer, and the underlying molecular mechanisms remain to be fully elucidated.

There are $>90$ types of protein tyrosine kinase in the human genome, 58 of which are receptor tyrosine kinases (RTK) 
containing a transmembrane domain, and they have been classified into 20 families (9). The TAM family, a subfamily of RTK, consists of three RTKs; Tyro3, also termed Sky; Axl, also termed Ark and Ufo; and Mer (10-13). These RTKs have similar extracellular regions, two immunoglobin-like domains and two fibronectin type III repeats, and a conserved kinase domain within the cytoplasmic region (14). The growth arrest-specific 6 (Gas 6) and protein S vitamin K-dependent proteins have been identified as the biological ligands, which bind to TAM RTKs and transduce a variety of intracellular downstream signals including those involved in cell proliferation and survival, inhibition of apoptosis, cell adhesion, cell morphology and motility $(15,16)$. TAM receptors have been demonstrated as being important in normal cellular function involved in the initiation and progression of several types of cancer (17). Notably, the protein levels of Axl, Mer and/or Gas 6 have been reported to be elevated in numerous cancer cell lines and patient samples, including leukemia $(18,19)$, multiple myeloma $(20)$, breast cancer $(21,22)$, colon cancer $(23)$, gastric cancer (24), liver cancer (25), melanoma (26), ovarian cancer (27), prostate cancer (28) and renal cell carcinoma (29). Therefore, these TAM RTKs and their ligands may be reliable biomarkers for the diagnosis and prognosis of various types of cancer and offer potential targets for the their treatment.

In the present study, SKOV3/TR taxol-resistant ovarian cancer cells were established to investigate the biological effects of TAM RTKs and the mechanisms underlying the development of resistance, which may serve as possible interventions to restore the chemosensitivity of these resistant cells.

\section{Materials and methods}

Reagents and antibodies. Primers for Ax1, Tyro3, Mer and GAPDH were synthesized from Bioneer, Inc. (Daejoun, Korea). TRIzol reagent was obtained from Solgent (Daejoun, Korea). AmpliTaq DNA polymerase was obtained from Roche diagnostics (Indianapolis, IN, USA). For western blot analysis, 10X Tris-buffered saline with Tween-20 (TBST; cat. no. sc-24953) and specific antibodies against phosphorylated (phospho)-Akt (cat. no. sc-7985-R; anti-rabbit), Akt (cat. no. sc-8312; anti-rabbit), Axl (cat. no. sc-1096; anti-goat), phospho-signal transducers and activators of transcription 3 (STAT3; cat. no. sc-8059; anti-mouse), STAT3 (cat. no. sc-482; anti-rabbit), Tyro3 (cat. no. sc-1095; anti-goat), GAPDH (cat. no. sc-25778; anti-rabbit) and secondary antibodies (goat anti-rabbit IgG-HRP, cat. no. sc-2004; rabbit anti-goat IgG-HRP, cat. no. sc-2768; goat anti-mouse IgG-HRP; cat. no. sc-2005) were obtained from Santa Cruz Biotechnology Inc. (Dallas, TX, USA). Taxol (paclitaxel; cat. no. T7402) and NAC (cat no. A7250), were purchased from Sigma-Aldrich (St. Louis, MO, USA).

Cell culture. The SKOV3 cells were purchased from American Type Culture Collection and grown in RPMI-1640 (Gibco-BRL, Grand Island, NY, USA), containing 10\% FBS, $2 \mathrm{mM}$ L-glutamine, $10 \mathrm{U} / \mathrm{ml}$ penicillin and $10 \mathrm{~g} / \mathrm{ml}$ streptomycin, at $37^{\circ} \mathrm{C}$ in $5 \% \mathrm{CO}_{2}$ in a humidified atmosphere.

Establishment of taxol-resistant cells. The SKOV3/TR taxol-resistant ovarian cancer cells, which are a variant of
SKOV3, were established by stepwise exposure of the SKOV3 cells to increasing concentrations of taxol, ranging between 1.5 and $24 \mathrm{nM}$ over 6 months.

Reverse transcription-polymerase chain reaction (RT-PCR). The cells $\left(3 \times 10^{5}\right)$ were seeded into a $60 \mathrm{~mm}$ culture dish and grown overnight at $37^{\circ} \mathrm{C}$. Total RNA was extracted using TRIzol reagent and subjected to cDNA synthesis and RT-qPCR. Briefly, $2 \mu \mathrm{g}$ total RNA was reverse transcribed into cDNA using SuperScript First-Strand Synthesis System (Invitrogen Life Technologies, Carlsbad, CA, USA), according to the manufacturer's instructions. Using specific primers, the obtained cDNAs were amplified using a TGradient Thermal Cycler (Biometra, Goettingen, Germany) and AccuPower PCR PremixTM (Bioneer, Daejeon, Korea), according to the manufacturer's instructions. The cycling conditions were as follows: 3 min denaturation at $94^{\circ} \mathrm{C}, 30$ cycles of denaturation at $94^{\circ} \mathrm{C}$ for $30 \mathrm{sec}$, annealing at $55^{\circ} \mathrm{C}$ for $1 \mathrm{~min}$ and extension at $72^{\circ} \mathrm{C}$ for $30 \mathrm{sec}$, followed by a final cycle of $94^{\circ} \mathrm{C}$ for $30 \mathrm{sec}$ and $60^{\circ} \mathrm{C}$ for $5 \mathrm{~min}$. The specific primers were as follows: Axl, sense 5'-AACCTTCAACTCCTGCCTTCTCG-3' and antisense 5'-CAGCTTCTCCTTCAGCTCTTCAC-3'; Tyro3, sense 5'-GTGTGTGGCTGACTTCGGAC-3' and antisense 5'-CACGTCCTCCATACACTCCG-3'; Mer, sense 5'-CGACTAAGCAGCAGGATGGA-3' and anti-sense 5'-GAGGGGGCATAATCTACCCA-3'; and GAPDH, sense 5'-GGAGCCAAAAGGGTCATCAT-3' and antisense 5'-GTGATGGCATGGACTGTGGT-3'.

Western blot analysis. The cells were treated with $\mathrm{N}$-acetyl cysteine (NAC) at $37^{\circ} \mathrm{C}$ for $24 \mathrm{~h}$. The whole cell lysates were prepared from the cells using lysis buffer, containing $1 \%$ Triton $\mathrm{X}-100,50 \mathrm{mM}$ Tris (pH 8.0), $150 \mathrm{mM} \mathrm{NaCl}, 1 \mathrm{mM}$ phenylmethylsulfonyl fluoride, $1 \mathrm{mM} \mathrm{Na} \mathrm{VO}_{4}$, and protease inhibitor cocktail. The protein concentrations were determined using a Bio-Rad protein assay kit II (cat. no. 500-0002; Bio-rad Laboratories, Inc., Hercules, CA, USA). The proteins from the whole cell lysates $(20 \mu \mathrm{g}$ for Axl and Tyro3 detection or $40 \mu \mathrm{g}$ for p-Akt, Akt, p-STAT3 and STAT3 detection) were separated using $12 \%$ SDS-PAGE, and electrotransferred onto nitrocellulose membranes. The membranes were blocked for $30 \mathrm{~min}$ at room temperature in Tris-buffered saline with $0.05 \%$ Tween-20 (TTBS), containing 5\% non-fat dry milk, and incubated with TTBS containing primary antibody (1:1,000 dilution) for $4 \mathrm{~h}$ at room temperature. Following three 10 min washes in TTBS, the membranes were incubated with peroxidase-conjugated secondary antibody (1:3,000 dilution) for $1 \mathrm{~h}$. Following three additional $10 \mathrm{~min}$ washes with TTBS, the protein bands were visualized using an enhanced chemiluminescence detection system $\left(\right.$ Amersham ${ }^{\mathrm{TM}} \mathrm{ECL}^{\mathrm{TM}}$ Prime Western Blotting Detection reagent; GE Healthcare, Piscataway, NJ, USA). Images of protein bands were captured using an LAS-3000 Image Analysis system (FujiFilm, Tokyo, Japan) and the relative quantities of each protein band were analyzed using Image Gauge 3.01 software (Fuji Photofilm; Fujifilm).

Colony-forming assay. The cells ( 1 to $2 \times 10^{3} /$ dish) were seeded into $35 \mathrm{~mm}$ culture dishes and allowed to grow for between 7 days (for untreated cells) and 10 days (for experiments 
using siRNA or NAC) to form colonies. Colonies containing $>50$ cells were visualized by crystal violet staining (in $60 \%$ methanol; Junsei Chemical Co, Ltd., Tokyo, Japan). Images of the cells were captured using an LAS 3000 Image Analysis system (FujiFilm, Tokyo, Japan).

Cell viability assay. The viability of the cells was measured using a Cell Counting Kit-8 assay kit (Dojindo Laboratories, Kumamoto, Japan). The cells $\left(1 \times 10^{3}\right.$ cells/well) were seeded into 96 -well plates and grown overnight at $37^{\circ} \mathrm{C}$. The cells were subsequently incubated at $37^{\circ} \mathrm{C}$ for 24,48 and $72 \mathrm{~h}$ to measure cell proliferation rate or treated with $0.001,10,100$, and 1,000 $\mathrm{nM}$ taxol for $72 \mathrm{~h}$ to observe the acquisition of taxol resistance. Following treatment, $10 \mu \mathrm{l}$ CCK- 8 solution was added and the cells were incubated for $4 \mathrm{~h}$. The absorbance at $450 \mathrm{~nm}$ was measured using a microplate reader (Model 680 microplate reader; Bio-Rad Laboratories, Inc.). The values are expressed as the mean \pm standard deviation for triplicate wells, and normalized to that of the control group to determine the percentage viability.

Small interfering (si)RNA transfection. RNA interference silencing was performed to decrease the protein level of Tyro3. The SKOV3 or SKVO3/TR cells $\left(1 \times 10^{6}\right)$ were seeded into $100 \mathrm{~mm}$ culture dishes and grown overnight. The cells were then transfected with $50 \mathrm{nM}$ siRNA against Tyro3 (cat. no. sc-36438; Santa Cruz Biotechnology, Inc.), or control siRNA (cat. no. sc-37007; Santa Cruz Biotechnology, Inc.). For the cell proliferation and colony formation assays, the cells were harvested $24 \mathrm{~h}$ after transfection and were reseeded into $35 \mathrm{~mm}$ culture dishes and allowed to grow for 10 days. The cells were harvested $48 \mathrm{~h}$ after transfection and western blot analysis were performed to evaluate the protein levels of Tyro3.

ROS measurement. Fluorescence-activated cell sorting analysis was performed to determine the intracellular levels of ROS. The cells $\left(1 \times 10^{6}\right)$ were seeded into $100 \mathrm{~mm}$ culture dishes and grown overnight. The cells were then stained with $5 \mathrm{mg} / \mu 12$ ', 7'-dichlorodihydrofluorescin diacetate for $30 \mathrm{~min}$ and subjected to flow cytometry using a Becton-Dickinson FACSCalibur (Becton-Dickinson, San Jose, CA, USA) and analyzed using CellQuest software (Becton-Dickinson).

Statistical analysis. All data are expressed as the mean \pm standard deviation of triplicate samples or at least three independent experiments. Student's t-test was used to determine statistical significance and $\mathrm{P}<0.05$ was considered to indicate a statistically significant difference.

\section{Results}

Long-term exposure of ovarian cancer cells to taxol results in taxol resistance and a reduction in proliferation capacity. To characterize and investigate the underlying mechanisms of chemoresistance in ovarian cancer cells, taxol-resistant SKOV3/TR cells were established by stepwise long-term exposure of the SKOV3 cells to gradually increasing concentrations of taxol over 6 months. As shown in Fig. 1A, the SKOV3/TR cells were found to be significantly smaller in size and more rounded in shape compared with the parental
SKOV3 cells, indicating that the SKOV3/TR cells exhibited different morphological features.

The acquisition of taxol resistance was evaluated by comparing the viability of the parental SKOV3 cells and SKOV3/TR cells following treatment with taxol at concentrations ranging between 0.01 and $1,000 \mu \mathrm{M}$. The cell viability was determined using a CCK- 8 assay. Taxol treatment decreased the proliferation of the parental SKOV3 cells and the variant SKOV3/TR cells in dose-dependent manner. However, at concentrations of taxol $>10 \mu \mathrm{M}$, the SKOV3 cells were significantly more sensitive to taxol treatment compared with the SKOV3/TR cells, suggesting the acquisition of taxol resistance (Fig. 1B).

The present study also found that proliferation rate of the SKOV3/TR cells was decreased compared with that of the parental SKOV3 cells (Fig. 1C), indicating the prolonged population doubling time (PDT) of the SKOV3/TR cells. The results of the colony-forming assay further demonstrated the prolonged PDT of the SKOV3/TR cells, since the SKOV3/ TR cells formed significantly fewer colonies, compared with the SKOV3 cells (Fig. 1D). In addition, the sizes of the single colonies formed by the SKOV3/TR cells were smaller than that formed by the SKOV3 cells, indicating a smaller number of SKOV3/TR cells compared with the SKOV3 cells. Taken together, these results indicated that taxol resistance had developed in the SKOV3/TR, with a concomitant reduction in proliferative capacity.

Expression of Tyro3 RTK is upregulated in taxol-resistant cells. Since the TMA family of RTKs has been reported as being important on cell survival and proliferation (30), the present study examined whether the acquisition of taxol resistance affected the mRNA and protein expression levels of TAM RTKs. Tyro3, Axl, and Mer in the SKOV3 and SKOV3/TR cells. The RT-qPCR results revealed that the mRNA expression of Tyro3 in the SKOV3/TR cells was increased compared to that of the parental SKOV3 cells, while the mRNA levels of Axl and Mer were decreased (Fig. 2A). The upregulation of Tyro3 in the SKOV3/TR cells was further confirmed using western blot analysis. As shown in Fig. 2B, the protein levels of Tyro3 and Axl in the SKOV3/TR cells were higher and lower than those in the parental SKOV3 cells, respectively, consistent with the RT-qPCR results. Of note, these results suggested that, among the TAM RTKs, only the expression of Tyro3 was upregulated and responsible for the acquisition of taxol resistance in the SKOV3/TR cells.

Tyro3 specific siRNA transfection into taxol-resistant cells suppresses cell proliferation. The present study also examined the effect of overexpression of the Tyro3 protein on the proliferation of the SKOV3/TR cells. The SKOV3/TR cells were transfected with siTyro3 and their viability was determined. Western blot analysis demonstrated that transfection with siTyro3, but not control siRNA, into the SKOV3/TR cells significantly decreased the protein expression of Tyro3 (Fig. 2C). The effect of siTyro3 on cell proliferation was determined using a colony-forming assay. As shown in Fig. 2D, the SKOV3/TR cells transfected with siTyro3 formed fewer colonies compared with the same cells transfected with siCtrl (Fig. 2D). Taken together, these results demonstrated that 
A

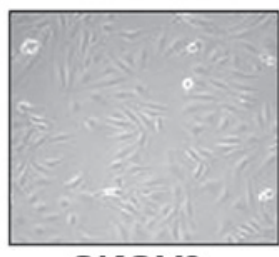

SKOV3

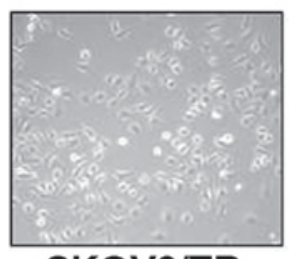

SKOV3/TR
C

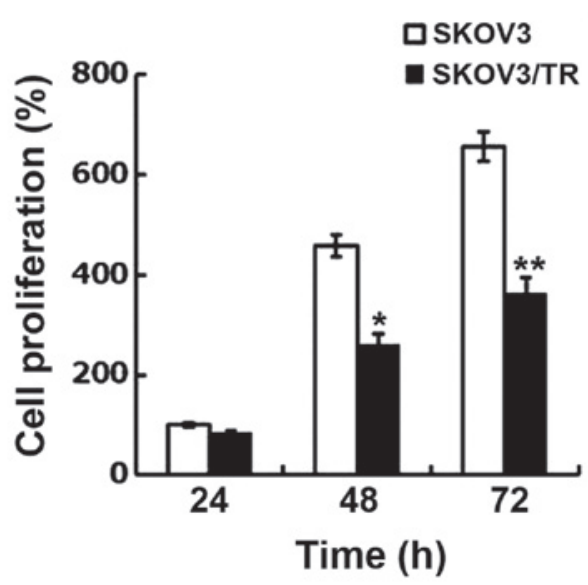

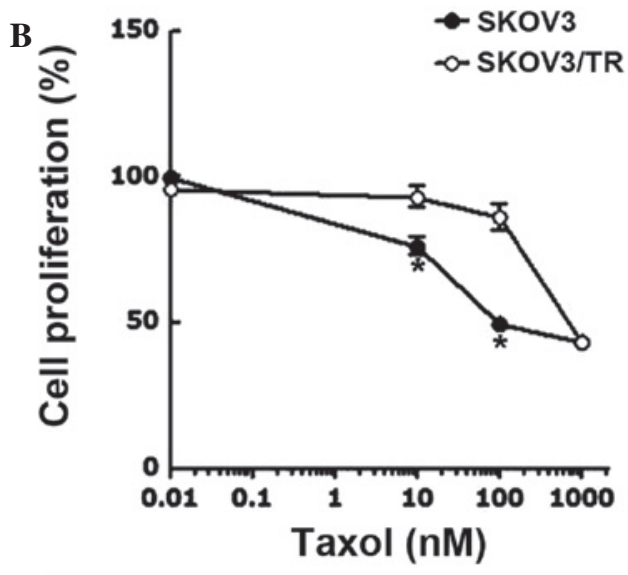

D
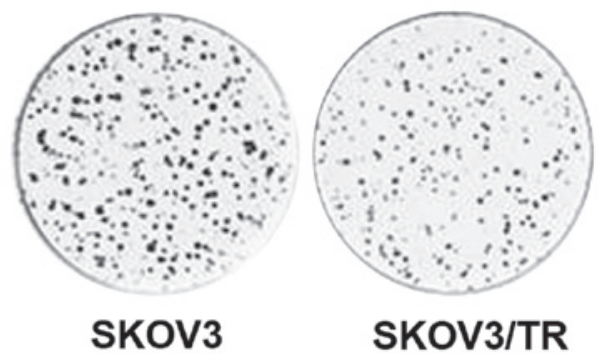

Figure 1. Development taxol resistance causes morphological changes and reduces the proliferative capability of taxol-resistant ovarian cancer cells. SKOV3 cells were exposed to increasing concentrations of taxol in a stepwise manner, which resulted in the emergence of taxol-resistant SKOV3/TR cells. (A) Under a light microscope (an inverted Nikon phase contrast microscope; magnification, x200), the morphological characteristics of each cell differed. (B) Parental SKOV3 and taxol-resistant SKOV3/TR cells were treated with 0.01- 1,000 mM taxol for 24 h. Cell viability was measured using a CCK-8 assay. The data are expressed as the mean \pm standard deviation of at least three replicates. ( ${ }^{*} \mathrm{P}<0.05 \mathrm{SKOV} 3$, vs. SKOV3/TR at 10 and $100 \mu \mathrm{M}$ taxol). (C) Cells $\left(1 \mathrm{x} 10^{3}\right.$ cells/well) were seeded onto 96-well plates and allowed to grow for the indicated periods. The proliferation rate was determined using a CCK-8 assay. Data are expressed as the mean \pm standard deviation of three independent experiments. ( ${ }^{*} \mathrm{P}<0.05$ SKOV3, vs. SKOV3/TR at $48 \mathrm{~h}$; ${ }^{* *} \mathrm{P}<0.001 \mathrm{SKOV} 3$, vs. SKOV3/TR at $72 \mathrm{~h}$ ). (D) Cells $\left(2 \times 10^{3}\right.$ cells/dish) were seeded into $35 \mathrm{~mm}$ dishes and allowed to grow for 7 days (untreated) or 10 days (treated) to form colonies. The colonies were visualized using crystal violet staining and their images were captured using an LAS-3000 Image Analysis system. The data is representative of at least three independent experiments. CCK-8, cell-counting-kit 8.

upregulation of the expression of Tyro3 was responsible for the proliferation of SKOV3/TR cells, eventually causing the acquisition of taxol resistance.

Anti-oxidant NAC reduces the proliferation of taxol-resistant cells via downregulation of the expression of Tyro3 and inhibiting the phosphorylation of Akt. The production of ROS banticancer drugs is a well-known and essential mechanism to induce apoptosis, which in turn, abrogate the proliferation of cancer cells $(31,32)$. Therefore, the present study assessed the involvement of ROS in the acquired taxol resistance of SKOV3/TR cells. FACS analysis was performed to measure ROS contents of SKOV3 and SKOV3/TR cells. As shown in Fig. 3A, the intracellular ROS content of SKOV3/TR cells was higher than that of the parental cells. To investigate the biological relevance of the increased level of intracellular ROS in the SKOV3/TR cells the effect of the NAC antioxidant reagent on the proliferation of SKOV3 and SKOV3/TR cells was examined.. The cells were treated with 5,10 or $20 \mathrm{mM}$
NAC for 24 , following which the viable cell numbers were counted. The results demonstrated that the viability of the SKOV3/TR cells treated with $10 \mathrm{mM}$ NAC was slightly reduced (21\% reduction), while that of the SKOV3 cells was unaffected by this dose of NAC. However, at $20 \mathrm{mM}$ NAC, the viability of each cell was reduced to $69 \%$ (SKOV3 cell) and 50\% (SKOV3/TR cells), indicating that SKOV3/TR cells were more sensitive to NAC treatment compared with the parental cells (Fig. 3B). This profound antiproliferative effect of NAC on the SKOV3/TR cells was further verified using a colony-forming assay. As shown in Fig. 3C, the SKOV3/TR cells were found to form significantly smaller colony sizes and fewer colonies in the presence of $20 \mathrm{mM}$ NAC compared with the parental cells. These results indicated that the elevated ROS in taxol-resistant SKOV3/TR cells was responsible for the proliferation of the cells.

As a reduction in the protein level of Tyro3 by Tyro-specific siRNA had antiproliferative effects in the SKOV3/TR cells, whether the antiproliferative effect of NAC also results from 
A

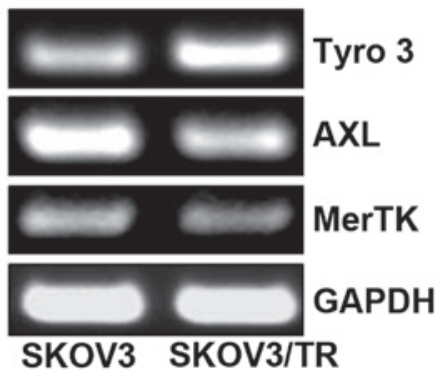

C

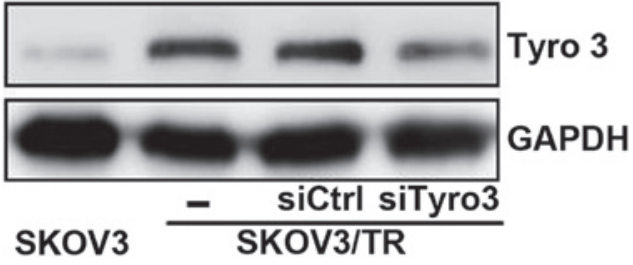

B

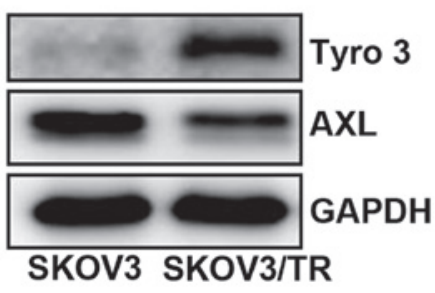

D

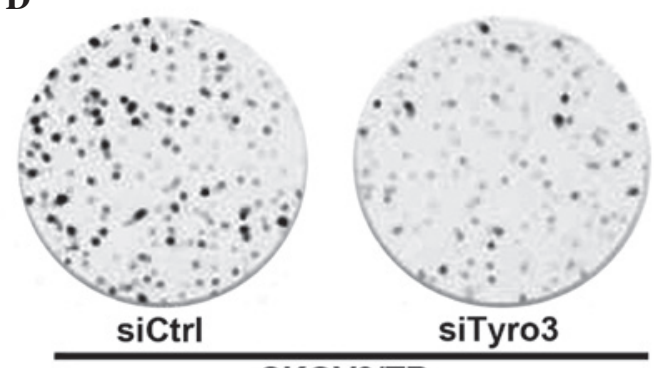

SKOV3/TR

Figure 2. Upregulation of the expression of Tyro3 leads to the proliferation of taxol-resistant ovarian cancer cells. (A) Total RNA from the SKOV3 and SKOV3/TR cells were isolated and used for RT-PCR to analyze the mRNA expression levels of Axl, Tyro3, and Mer. As an internal control, GAPDH mRNA was also amplified by RT-PCR. The data are representative of three independent experiments. (B) Protein levels of Axl and Tyro3 in the SKOV3 and SKOV3/TR cells was determined using western blot analysis. GAPDH was used as a loading control. The results are representative of three independent

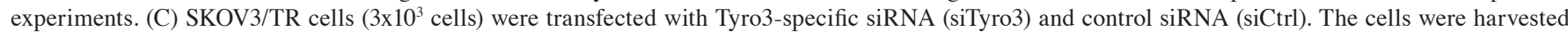
$48 \mathrm{~h}$ after transfection, and the protein level of Tyro3 was determined using western blot analysis. GAPDH was used as a loading control. The results are representative of three independent experiments. (D) Cells transfected with siTyro3 or siCtrl were harvested $24 \mathrm{~h}$ after transfection and each cell (2x10 3 cells/ dish) was seeded into $35 \mathrm{~mm}$ dishes and grow for 7-10 days. The colonies were visualized using crystal violet staining. The result is a representative of at least three independent experiments. RT-PCR, reverse transcription quantitative polymerase chain reaction. siRNA, small interfering RNA.

modulation of the expression of Tyro3 was examined. The cells were treated with $20 \mathrm{mM}$ NAC and the protein levels of Tyro3 were determined using western blot analysis. As shown in Fig. 4A, the expression of Tyro3 was inhibited by NAC, confirming that the elevated level of intracellular ROSinduced the expression of Tyro3, which subsequently resulted in proliferation of the SKOV3/TR cells Western blot analysis further revealed the inhibitory effect of NAC on Akt phosphorylation, which transduces proliferative signal and was increased in the SKOV3/TR cells (Fig. 4B). However, NAC treatment had no effect on the phosphorylation or the induction of STAT3, a well-known positive regulator of cell proliferation (Fig. 4C). Taken together, these results indicate that upregulation of the expression of Tyro3 ant phosphorylation of Akt, but not the phosphorylation or induction of STAT3, were associated with the increased intracellular ROS contents in the SKOV3/TR cells, resulting in proliferation of the cells.

\section{Discussion}

Ovarian cancer is the first leading cause of death among gynecological cancers (33). The majority of patients with ovarian cancer are confronted with refractoriness against standard chemotherapy, a combination of platinum, containing cisplatin, carboplatin and paclitaxel, and develop further resistance to subsequent treatments (1). Therefore, the characterization and understanding of the molecular mechanisms associated with chemoresistance is valuable in overcoming cases of ovarian cancer relapse.

The present study developed SKOV3/TR taxol-resistant ovarian cancer cells, which were found to exhibit a reduction in size and slower growth rates compared with the parental SKOV cells. While the prolongedPDT of the taxol-resistantSKOV3/TR cells was consistent with a phenotype of therapy-induced senescence (TIS), their morphological characteristics of a reduced size and increased roundedness, compared with the parental cells, is opposite to those observed in TIS (34), since the morphology of senescent cells is enlarged and flattened. It may be beneficial to examine wether SKOV3/TR cells have other characteristics similar to TIS, including the activation of senescence-associated $\beta$-galactosidase, the development of senescence-associated heterochromatin foci or the secretion of excessive tumor-promoting cytokines, as TIS has inhibitory effects on proliferation and apoptosis, and senescent cancer cells require elimination for the successful treatment of cancer.

Resistance to tyrosine kinase inhibitors, including imatinib and nilotinib, has been correlated with the activation and overexpression of TAM RTKs in chronic myeloid leukemia cells $(35,36)$. Consistent with the these reports, the present study demonstrated that the expression of Tyro3 RTK was elevated in the taxol-resistant SKOV3/TR cells, and knockdown of Tyro3 using specific siRNA sensitized the SKOV3/TR cells to taxol. However, the expression levels of the other TAM RTKs, Axl and Mer, were inversely downregulated in these 
A

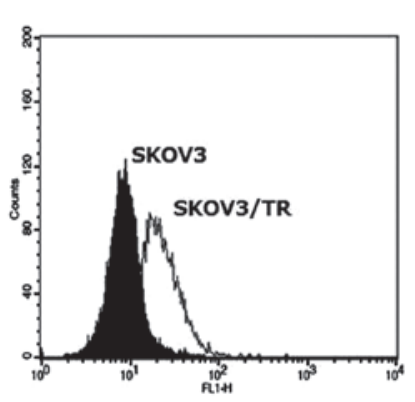

C
B

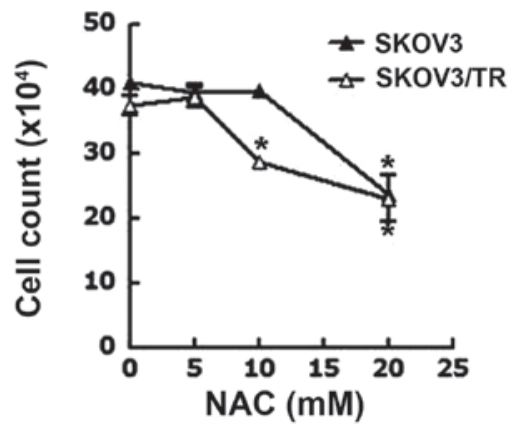

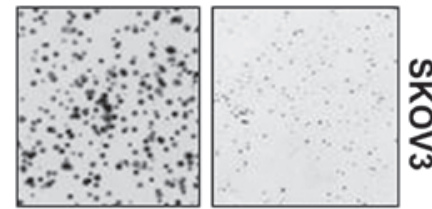
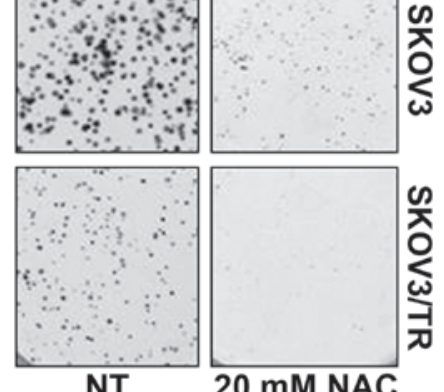

NT

20 mM NAC

Figure 3. Antioxidant NAC inhibits the proliferation of taxol-resistant ovarian cancer cells. (A) To determine the level of intracellular ROS, fluorescence-activated cell sorting analysis was performed. The cells $\left(3 \times 10^{3} \mathrm{cells} / \mathrm{dish}\right)$ were seeded ointo $100 \mathrm{~mm}$ dishes, grown overnight and stained with DCF-DA using flow cytometry. The result shown is representative of three independent experiments. (B) Cells ( $\left.3 \times 10^{3} \mathrm{cells} / \mathrm{dish}\right)$ were seeded into $100 \mathrm{~mm}$ dishes, grown overnight and treated with the indicated concentrations of NAC for $24 \mathrm{~h}$. The number of viable cells was counted. Data are expressed as the mean \pm standard deviation of three independent experiments. ("P $<0.05$, SKOV3 vs. SKOV3/TR at $10 \mathrm{mM} \mathrm{NAC}$ and untreated, vs. $20 \mathrm{mM}$ NAC). (C) Cells ( $2 \times 10^{3}$ cells/dish) were seeded onto $35 \mathrm{~mm}$ dishes and treated with $20 \mathrm{mM}$ NAC. After $24 \mathrm{~h}$, the cells were washed with phosphate-buffered saline and allowed to grow for 7-10 days. The colonies were visualized using crystal violet staining. The results shown are representative of three independent experiments. NAC, N-acetyl cysteine; NT, untreated.

A
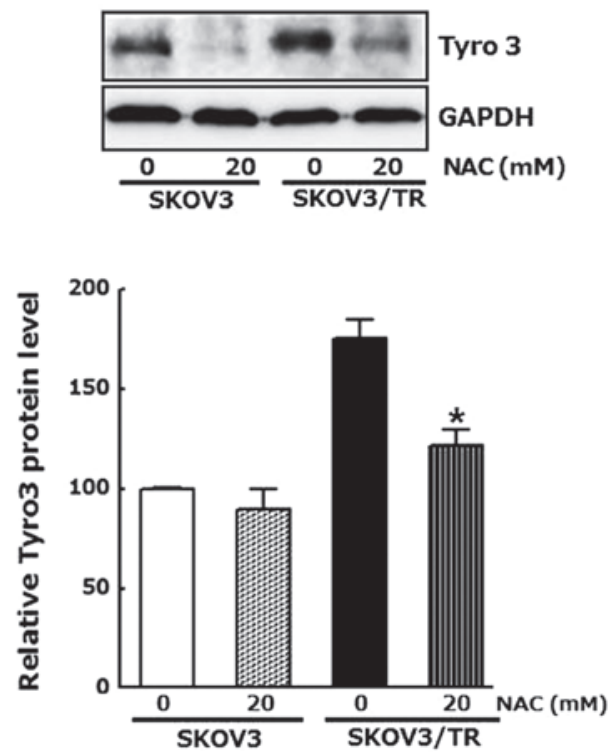

B

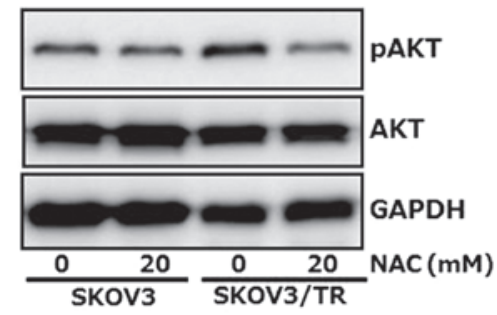

C

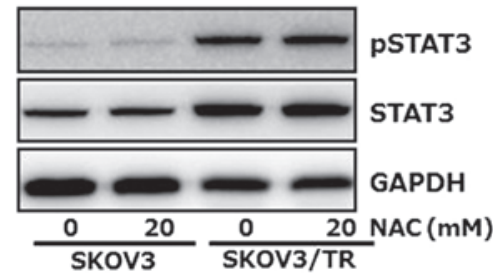

Figure 4. Antioxidant NAC suppresses the expression of Tyro3 RTKs and phosphorylation of Akt in taxol-resistant ovarian cancer cells. Cells ( $3 \times 10^{3}$ cells/dish) were seeded into $100 \mathrm{~mm}$ dishes, grown overnight and treated with $20 \mu \mathrm{M}$ NAC for $24 \mathrm{~h}$, following which the total cell lysates were prepared. (A) Protein expression of Tyro3 was determined using western blot analysis to determine the effect of NAC on its expression. The relative quantity of Tyro3 protein was calculated using Image Gauge 3.01 software. The data are expressed as the mean \pm standard deviation of three independent experiments. (" $\mathrm{P}<0.05$,

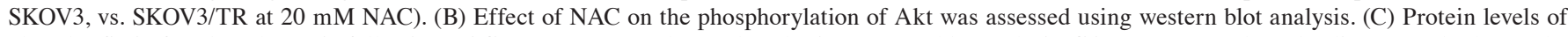
phospho-STAT3 and total protein following NAC treatment were also analyzed using western blot analysis. GAPDH was used as a loading control. The results shown are representative of three independent experiments. NAC, N-acetyl cysteine; STAT3, signal transducers and activators of transcription 3; pSTAT3, phosphorylated STAT3. 
cells, indicating that the increased expression of Tyro3 plays is important in the survival and proliferation of SKOV3/TR cells, while decreased levels of Axl and Mer may be responsible for their prolonged PDT.

One of the antiproliferative mechanisms of chemotherapeutic drugs is the induction of ROS, which leads to apoptosis of cancer cells (31). The role of ROS in developing chemoresistance is complex, as elevation and reduction are observed in the levels of ROS during the acquisition of drug resistance (37). The present study found that, in the SKOV3/TR cells, the intracellular ROS contents were increased and their proliferation was suppressed by NAC treatment. The correlation between the production of ROS and the development of taxol resistance in the SKOV3/TR cells was further confirmed by western blot analysis, which demonstrated the inhibitory effect of NAC on the expresion of Tyro3. Taken together, these results suggested that the levels of ROS were increased during the acquisition of taxol resistance in the SKOV3/TR cells, which upregulated the expression of Tyro3, and concomitantly led to the survival and proliferation of these cells via overexpression of the Tyro 3 protein.

The present study also demonstrated that NAC treatment reduced the level of phospho-Akt, which was increased in the SKOV3/TR cells, suggesting that Tyro3 signaled through the Akt pathway to promote cell proliferation. This result was consistent with that of a previous study, which reported that phosphoinositide-3-kinase/Akt pathway is linked to the activation of Tyro3 (38). However, NAC did not affect the phosphorylation or protein levels of STAT3, indicating that the increased phosphorylation and expression of STAT3 in the SKOV3/TR cells may not be associated with the elevated level of intracellular ROS in the cells.

In conclusion, the present study demonstrated that long-term exposure of human ovarian cancer SKOV3 cells resulted in the formation of taxol-resistant SKOV3/TR cells via the induction of intracellular ROS, subsequent upregulation in the expression of Tyro3 and the activation of Akt, which promoted the survival and proliferation of the resistant cells. Thus, modulation of the expression of Tyro3 and/or its role in the downstream signaling pathway may offer a potential target in overcoming taxol resistance.

\section{Acknowledgements}

This study was supported by the Basic Science Research Program through the National Research Foundation of Korea, funded by the Ministry of Education, Science and Technology (grant. no. 2006-2005303).

\section{References}

1. Agarwal R and Kaye SB: Ovarian cancer: strategies for overcoming resistance to chemotherapy. Nat Rev Cancer 3: 502-516, 2003.

2. Reed E, Kohn EC, Sarosy G, et al: M Paclitaxel, cisplatin, and cyclophosphamide in human ovarian cancer: molecular rationale and early clinical results. Semin Oncol 22(3 Suppl 6): 90-96, 1995.

3. Raja FA, Counsell N, Colombo N, et al: Platinum versus platinumcombination chemotherapy in platinum-sensitive recurrent ovarian cancer: a meta-analysis using individual patient data. Ann Oncol 24(12):3028-3034, 2013.

4. Einzig AI, Wiernik PH, Sasloff J, Runowicz CD and Goldberg GL: Phase II study and long-term follow-up of patients treated with taxol for advanced ovarian adenocarcinoma. J Clin Oncol 10 $1748-1753,1992$
5. Tian C, Ambrosone CB, Darcy KM, et al: Common variants in ABCB1, ABCC2 and ABCG2 genes and clinical outcomes among women with advanced stage ovarian cancer treated with platinum and taxane-based chemotherapy: a Gynecologic Oncology Group study. Gynecol Oncol 124: 575-581, 2012.

6. Cannistra SA: Cancer of the ovary. N Engl J Med 351: 2519-2529, 2004.

7. McGuire WP 3rd: Current status of taxane and platinum-based chemotherapy in ovarian cancer. J Clin Oncol 21 (Suppl 10): 133-135, 2003

8. Ozols RF: Systemic therapy for ovarian cancer: current status and new treatments. Semin Oncol 33: 3-11, 2006.

9. Robinson DR, Wu YM and Lin SF: The protein tyrosine kinase family of the human genome. Oncogene 19: 5548-5557, 2000

10. Ohashi K, Mizuno K, Kuma K, Miyata T and Nakamura T: Cloning of the cDNA for a novel receptor tyrosine kinase, Sky, predominantly expressed in brain. Oncogene 9: 699-705, 1994.

11. O'Bryan JP, Frye RA, Cogswell PC, et al: axl, a transforming gene isolated from primary human myeloid leukemia cells, encodes a novel receptor tyrosine kinase. Mol Cell Biol 11: 5016-5031, 1991.

12. Janssen JW, Schulz AS, Steenvoorden AC, et al: A novel putative tyrosine kinase receptor with oncogenic potential. Oncogene 6: 2113-2120, 1991.

13. Rescigno J, Mansukhani A and Basilico C: A putative receptor tyrosine kinase with unique structural topology. Oncogene 6: 1909-1913, 1991.

14. Sasaki T, Knyazev PG, Clout NJ, et al: Structural basis for Gas6-Axl signalling. EMBO J 25: 80-87, 2006.

15. Stitt TN, Conn G, Gore M, et al: The anticoagulation factor protein $\mathrm{S}$ and its relative, Gas6, are ligands for the Tyro 3/Axl family of receptor tyrosine kinases. Cell 80: 661-670, 1995.

16. Hafizi S and Dahlbäck B: Gas6 and protein S. Vitamin K-dependent ligands for the Axl receptor tyrosine kinase subfamily. FEBS J 273: 5231-5244, 2006.

17. Zwick E, Bange $\mathbf{J}$ and Ullrich A: Receptor tyrosine kinase signalling as a target for cancer intervention strategies. Endocr Relat Cancer 8: 161-173, 2001.

18. Challier C, Uphoff CC, Janssen JW and Drexler HG: Differential expression of the ufo/axl oncogene in human leukemia-lymphoma cell lines. Leukemia 10: 781-787, 1996.

19. Graham DK, Salzberg DB, Kurtzberg J, et al: Ectopic expression of the proto-oncogene Mer in pediatric T-cell acute lymphoblastic leukemia. Clin Cancer Res 12: 2662-2669, 2006.

20. De Vos J, Couderc G, Tarte K, et al: Identifying intercellular signaling genes expressed in malignant plasma cells by using complementary DNA arrays. Blood 98: 771-780, 2001.

21. Meric F, Lee WP, Sahin A, Zhang H, Kung HJ and Hung MC: Expression profile of tyrosine kinases in breast cancer. Clin Cancer Res 8: 361-367, 2002.

22. Tavazoie SF, Alarcón C, Oskarsson T, et al: Endogenous human microRNAs that suppress breast cancer metastasis. Nature 451: 147-152, 2008

23. Craven RJ, Xu LH, Weiner TM, et al: Receptor tyrosine kinases expressed in metastatic colon cancer. Int J Cancer 60: 791-797, 1995.

24. Wu CW, Li AF, Chi CW, et al: Clinical significance of AXL kinase family in gastric cancer. Anticancer Res 22: 1071-1078, 2002.

25. Tsou AP, Wu KM, Tsen TY, et al: Parallel hybridization analysis of multiple protein kinase genes: identification of gene expression patterns characteristic of human hepatocellular carcinoma. Genomics 50: 331-340, 1998.

26. Györffy B and Lage H: A Web-based data warehouse on gene expression in human malignant melanoma. J Invest Dermatol 127: 394-399, 2007.

27. Macleod K, Mullen P, Sewell J, et al: Altered ErbB receptor signaling and gene expression in cisplatin-resistant ovarian cancer. Cancer Res 65: 6789-6800, 2005.

28. Sainaghi PP, Castello L, Bergamasco L, Galletti M, Bellosta P and Avanzi GC: Gas6 induces proliferation in prostate carcinoma cell lines expressing the Axl receptor. J Cell Physiol 204: 36-44, 2005.

29. Chung BI, Malkowicz SB, Nguyen TB, Libertino JA and McGarvey TW: Expression of the proto-oncogene Axl in renal cell carcinoma. DNA Cell Biol 22: 533-540, 2003.

30. van Ginkel PR, Gee RL, Shearer RL, et al: Expression of the receptor tyrosine kinase Axl promotes ocular melanoma cell survival. Cancer Res 64: 128-134, 2004. 
31. Ozben T: Oxidative stress and apoptosis: impact on cancer therapy. J Pharm Sci 96: 2181-2196, 2007.

32. Lau AT, Wang Y and Chiu JF: Reactive oxygen species: current knowledge and applications in cancer research and therapeutic. J Cell Biochem 104: 657-667, 2008.

33. Permuth-Wey J and Sellers TA: Epidemiology of ovarian cancer. Methods Mol Biol 472: 413-437, 2009.

34. Collado M and Serrano M: Senescence in tumours: evidence from mice and humans. Nat Rev Cancer 10: 51-57, 2010.

35. Gioia R, Leroy C, Drullion C, et al: Quantitative phosphoproteomics revealed interplay between Syk and Lyn in the resistance to nilotinib in chronic myeloid leukemia cells. Blood 118: 2211-2221, 2011.
36. Dufies M, Jacquel A, Belhacene N, et al: Mechanisms of AXL overexpression and function in Imatinib-resistant chronic myeloid leukemia cells. Oncotarget 2: 874-885, 2011.

37. Achuthan S, Santhoshkumar TR, Prabhakar J, Nair SA and Pillai MR: Drug-induced senescence generates chemoresistant stemlike cells with low reactive oxygen species. J Biol Chem 286: 37813-37829, 2011.

38. Zhong Z, Wang Y, Guo H, et al: Protein S protects neurons from excitotoxic injury by activating the TAM receptor Tyro3-phosphatidylinositol 3-kinase-Akt pathway through its sex hormone-binding globulin-like region. J Neurosci 30: $15521-15534,2010$ 